\title{
Caracterización de discontinuidades típicas en soldadura, utilizando la técnica de Ultrasonido Pulso Eco-Scan A
}

\author{
Characterization of typical defects in welding using \\ Ultrasonic technique Pulse Eco-Scan A. \\ Oscar Javier Araque de los Ríos ${ }^{1}$ \\ ${ }^{I}$ MSc. Profesor Departamento de Ingeniería Mecánica, Universidad de Ibagué, Ibagué, Colombia \\ oscar.araque@unibague.edu.co
}

\begin{abstract}
Resumen- El ultrasonido es una de las técnicas más utilizadas en la inspección de uniones soldadas en aplicaciones estructurales, mecánicas, agro industriales entre otras, el presente artículo, muestra los resultados obtenidos de la utilización de la técnica ultrasonido Scan A, en diferentes probetas soldadas mediante el proceso SMAW, inicialmente se presenta una argumentación respecto a las ventajas y desventajas de la utilización de este tipo de ensayo no destructivo y el principio teórico de la técnica, el propósito principal es la identificación de discontinuidades típicas en uniones soldadas como son las porosidades, grietas de solidificación e inclusiones de escoria, estas presentan un haz característico que permite la establecer los distintos tipos de defectos, se incluyen los resultados de las inspecciones ultrasónicas correspondientes a las imperfecciones detectadas.
\end{abstract}

Palabras clave - Ultrasonido, soldadura, grietas de solidificación, poros, discontinuidades, inclusión de escoria, inspección.

Abstract- Ultrasonic technique is used in the inspection of welded joints in structural applications, mechanical, industrial agriculture among others, this article shows the results of using the ultrasonic technique Scan $A$, in different specimens welded by SMAW process, initially presented an argument about the advantages and disadvantages of using this type of nondestructive testing and the underlying principle of the technique, the main purpose is to identify typical discontinuities in welded joints such as porosity, solidification cracks and slag inclusions, beam these have a characteristic that allows the identification of various types of defects, the results of the ultrasonic inspection corresponding to the detected imperfections are included.

Key Word - Ultrasound, weld, solidification cracks, pores, discontinuities, slag inclusions, inspection.

Fecha de Recepción: 30 de Julio de 2015

Fecha de Aceptación: 28 de Diciembre de 2015

\section{INTRODUCCIÓN}

Las crecientes exigencias frente al cumplimiento de estándares en los diversos procesos de producción ha dado como resultado el desarrollo de tecnologías cuyo objeto es el de inspeccionar los diferentes productos, garantizando una correcta conformación y calidad de los componentes. Para realizar esto, existen diversas técnicas las cuales se clasifican principalmente como, ensayos destructivos (ED) y los ensayos no destructivos (END).

El objetivo principal de las pruebas destructivas o también conocidas con ensayos destructivos es determinar cuantitativamente el valor de ciertas propiedades de los materiales, como resistencia mecánica, la tenacidad o la dureza. La ejecución de las pruebas destructivas involucra el daño del material, la destrucción de la probeta o la pieza empleada en la valoración correspondiente, por lo anterior se afirma que los ensayos destructivos son la aplicación de métodos físicos directos que alteran de forma permanente las propiedades físicas, mecánicas o dimensionales de un material, parte o componente sujeto a inspección [1]. Los ensayos no destructivos, END o pruebas no destructivas PND (NDT en inglés), constituyen un campo que se desarrolla con gran rapidez en la ingeniería. Técnicas como: digitalización de imágenes, radiografía por neutrones, electromagnetismo o emisión acústica, no muy conocidas años atrás, son hoy en día comunes y constituyen parte importante de las herramientas utilizadas para el mantenimiento de la integridad en las industrias que desean mantenerse en la vanguardia del mercado ofreciendo calidad en sus productos [2].

Algunos de estos END posibilitan caracterizar piezas y detectar defectos presentes sin necesidad de perturbar su estructura, uno de estos ensayos es el Ultrasonido. Por esta 
razón resulta importante comprender el funcionamiento y las técnicas desarrolladas para elaborar los ensayos ultrasónicos.

El ensayo de ultrasonido requiere de instrumentos y equipos especializados, por ello es importante reconocer los elementos necesarios para las pruebas y capacitarse en el manejo de estos equipos. Para ello se propone realizar los ensayos pertinentes a diferentes probetas donde sea posible la caracterización física y la detección de defectos presentes en su construcción. En el presente documentos analizaran los defectos ocasionados por el proceso de soldadura por arco con electrodo metálico revestido (SMAW) en algunos elementos de uniones soldadas más comunes de la industria. La técnica de ultrasonido presenta especial interés debido a la aplicación industrial actual, tanto en el área de mantenimiento como la evaluación de estructuras metálicas. La validación de los procedimientos para identificación de defectos se realiza utilizando la técnica de inspección por Rayos X.

\section{ULTRASONIDO}

Este método se basa en la medición de la propagación de ondas ultrasónicas en el medio que se quiere analizar se propagan a través de este, se reflejan, difractan y atenúan para poder localizar defectos superficiales, subsuperficiales e internos en la pieza, realizar mediciones de espesores reales, de espesores de películas protectoras, de recubrimientos y en la inspección de soldaduras; su funcionamiento se basa en la impedancia acústica que es el producto de la velocidad máxima de propagación del sonido y la densidad del material

El sonido está conformado por un conjunto de ondas mecánicas longitudinales producidas por la vibración de los objetos y propagadas a través de un medio elástico. Este tipo de ondas pueden estimular el oído humano y generar una sensación sonora.

Las características de una onda de sonido son [3]:

- Ciclo: Es el movimiento completo de la onda.

- Longitud: Es la distancia necesaria para completar un ciclo. Por lo general se denota como $\lambda$.

- Amplitud: Es el valor máximo en sentido positivo y negativo que alcanza la onda sonora.

- Frecuencia: Es el número de ciclos por unidad de tiempo.

\section{Ventajas y desventajas del ultrasonido.}

Según la Norma Técnica Colombiana NTC 2120, las ventajas y desventajas más relevantes del ultrasonido en comparación con las otras pruebas de inspección son [4]:

- Ventajas
- Puede ser aplicado a una gran gama de materiales y productos.

- Fuera de su origen como aplicación industrial, también es empleado en medicina, navegación, pesca, comunicaciones entre otros.

- La portabilidad es una de sus principales características.

- El poder configurar la sensibilidad de equipo le permite revisar con mayor eficacia materiales sensibles.

\section{- Desventajas}

- Costo de los equipos y sus accesorios.

- Alto nivel de capacitación del personal que realizara las pruebas.

- La necesidad de emplear varios palpadores cuando se revisas una pieza compleja.

- El equipo debe ser calibrado con piezas que permitan establecer valores de referencia para su uso.

En el método ultrasónico se utilizan instrumentos que transmiten ondas con ciertos intervalos de frecuencia. Todos los materiales tienden a actuar hasta cierto punto como un filtro al paso de la onda, atenuando o dispersándolo. Las ondas del sonido oscilan a una frecuencia específica, esto es, número de vibraciones o ciclos por segundo. El oído humano percibe hasta una frecuencia máxima de aproximadamente 20000 ciclos por segundo $(20 \mathrm{KHz})$, mientras la mayoría de aplicaciones ultrasónicas utilizan frecuencias entre 500000 y 10000000 ciclos por segundo $(500 \mathrm{KHz}$ a $10 \mathrm{MHz})$. Las frecuencias altas son menos eficientes en el aire y otros gases, en la mayoría de los líquidos y materiales de ingeniería viaja libremente; se aplican para detectar defectos como poros, fisuras, también para conocer las propiedades básicas de los líquidos y sólidos como la composición, estructura. El análisis de los materiales mediante ultrasonido se basa en el principio físico del movimiento de una onda acústica, sabido es que la onda acústica es afectada por el medio a través del cual viaja y se distinguen los siguientes tipos de onda:

- Onda longitudinal

- Onda transversal

- Onda superficial (Rayleigh)

Debido a esto ocurren cambios asociados con el paso de una onda sonora de alta frecuencia a través de un material en uno o más de los cuatro parámetros siguientes: tiempo de tránsito, atenuación, reflexión y frecuencia. Estos parámetros a menudo pueden estar correlacionados con los cambios de las propiedades físicas, dureza, módulo de elasticidad, densidad, homogeneidad, estructura y grano del material. [5]

Existe diversidad de transductores de ultrasonido que son generalmente etiquetados de acuerdo a su diseño o uso previsto. Por ejemplo, los transductores de contacto son utilizados para aplicarlos directamente a, o por contacto con, una superficie sólida para el análisis. Algunos transductores 
de contacto son nombrados de acuerdo a su diseño general, como un elemento doble, una matriz lineal o un transductor convexo [6]. En cuanto a los métodos utilizados para realizar el ensayo de ultrasonido estos dependen principalmente de la accesibilidad que se tenga a la pieza, estas técnicas son por inmersión y por contacto directo, el método por contacto directo es uno de las más utilizados consiste en que el transductor está colocado directamente en la pieza de ensayo teniendo solamente en medio un material acoplante para lograr que las inspecciones sean realizadas con una mayor precisión.

\section{Técnicas de ensayo}

Las técnicas utilizadas para efectuar el ensayo de ultrasonido dependen del tipo de procedimiento a desarrollar y de factores como el material, la forma, condiciones superficiales etc, entre las técnicas más usadas se encuentra [6]:

- Transmisión: En este tipo de técnica se utilizan dos transductores, donde uno es el transmisor de alta frecuencia y el otro es el receptor; este método es limitado ya que solamente ofrece información de la presencia de discontinuidades en la pieza las cuales reducen la propagación del sonido que llega al receptor.

- Pulso Eco: En esta técnica la energía del sonido que está siendo reflejada y reciba es convertida nuevamente en energía eléctrica gracias al elemento piezoeléctrico que posee el transductor; su principio es la implementación de la porción que se refleja del sonido para la evaluación y análisis de los defectos, un cabezal cumple la doble función de ser emisor y receptor, la energía recibida es más débil que la se emite por tal motivo no se debe operar sobre la base del sonido continuo si no que se deben emplear únicamente los impulsos del sonido, un impulso eléctrico de una duración no más de un milisegundo genera una onda ultrasónica análoga, el equipo de ultrasonido posee la capacidad para que el oscilador se encuentra listo para la recepción aun cuando se está propagando la onda anteriormente disipada. La onda penetra el material a analizar, como resultado de la presencia de una superficie limite, se presenta una reflexión total o parcial de dicha onda, mostrando el resultado en la interfaz del equipo de ultrasonido. Se debe tener en cuenta que no solo las superficies limites generan los ecos si no que la presencia de defectos también los generan [7].

La presentación de los resultados de la inspección en el equipo de ultrasonido puede realizarse de diversas formas entre las principales se encuentran:

- Scan A: Cuando una onda es emitida y seguidamente regresa de forma parcial debido a la presencia de puntos con imperfección interna o de la parte de atrás de la pared del material (eco de fondo), esta información es visualizada en la pantalla del equipo de ultrasonido, presentando la amplitud del pulso y la duración del retorno al transductor, tal como se muestra en la Figura 1.

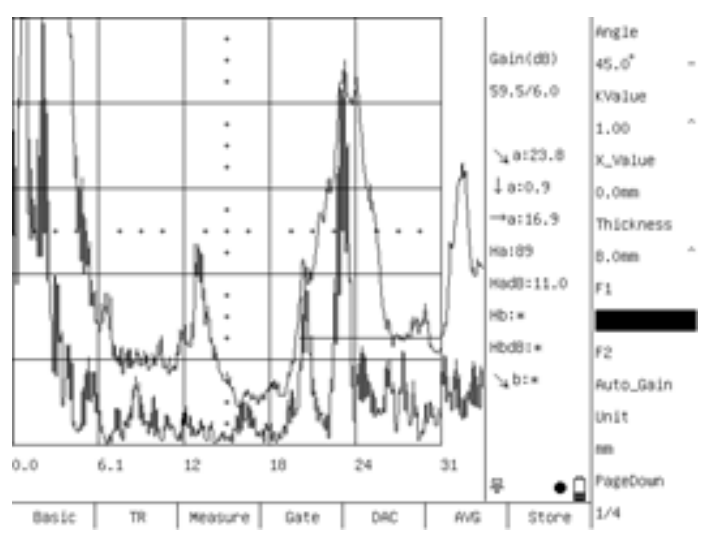

Figura 1. Imagen Scan A.

- Scan B: En este método de presentación gráfica los resultados de una serie de mediciones de espesor son representados por el perfil de la sección transversal del componente o pieza inspeccionada.

- Scan C: La presentación de la información se realiza de forma bidimensional, con un código de colores que representan la profundidad o espesor del material, objeto de estudio.

\section{Defectos en uniones soldadas}

La mayoría de los defectos en uniones soldadas son del tipo porosidad, falta de penetración, falta de fusión, inclusión de escoria, socavación y desalineamiento. El tamaño e intensidad de las imperfecciones depende del proceso de soldadura, la geometría, la facilidad de acceso, y el cuidado ejercido en el proceso de soldar, entre otros factores. Estas imperfecciones tienen diferentes características y en algunas situaciones son difíciles y costosas de detectar y definir sin destruir la junta soldada, varias de estas imperfecciones en soldaduras son mostradas en la Figura 2. [8].

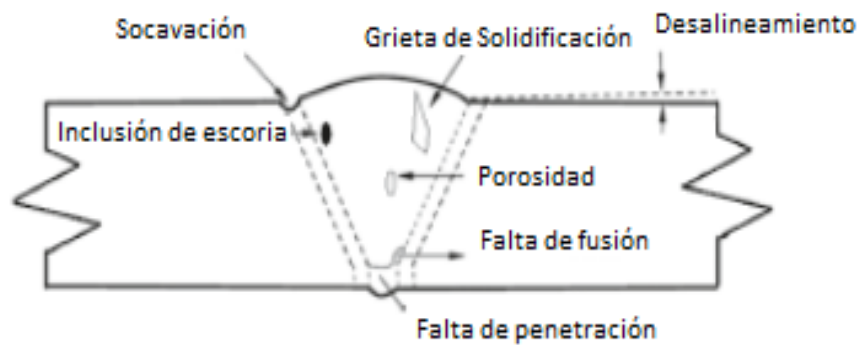

Figura 2. Imperfecciones de soldadura en juntas a tope. Adaptada de [7].

Existen códigos de aceptación para uniones soldadas en presencia de discontinuidades como poros, inclusiones y 
grietas o defectos de fusión. Para la aplicación de la inspección ultrasónica de materiales en servicio se considera que las grietas son defectos que se inician en condiciones de operación mientras que la porosidad, inclusiones, falta de fusión, etc. son formadas durante la fabricación. Algunos de los códigos ampliamente utilizados se enuncian a continuación.

- ANSI / ASME Boiler and Pressure Vessel Code (Código para Recipientes a Presión y Calderas de la Sociedad Americana de Ingenieros Mecánicos).

- ANSI / AWS D.1.1 Structural Welding Code (Código de Soldaduras Estructurales de la Sociedad Americana de Soldadura)

- ANSI / ASME B31 Piping Code (Código de tuberías de la Sociedad Americana de Ingenieros Mecánicos).

- ANSI / API 570 Piping Inspection Code (Código para Inspeccin de Tuberías delInstituto Americano del Petróleo)

\section{MATERIALES Y MÉTODOS}

Para realizar la caracterización de los diferentes defectos encontrados en las uniones soldadas objeto de estudio se utilizo un equipo de ultrasonido marca Olympus serie Epoch $\mathrm{XT}$, de igual forma un palpador angular de $45^{\circ}$ y frecuencia de $5 \mathrm{MHz}$, para el procedimiento de calibración inicial y de la sensibilidad se utilizo un bloque de calibración IIW Type I.

Las mediciones experimentales se realizaron en testigos no normalizados con geometrías diversas, todos ellos fabricados con material base acero ASTM A 36 de $9 \mathrm{~mm}$ de espesor y utilizando la técnica de soldadura por arco con electrodo metálico revestido (SMAW), el equipo utilizado para el depósito de la soldadura es marca Lincoln serie $135 \mathrm{~S}$ y electrodo E6013, las características eléctricas al momento del depósito son 73 voltios, $100 \mathrm{~A} \mathrm{CD}$.

En la figura 3, se puede observar el equipo de ultrasonido utilizado y las probetas objeto de estudio.

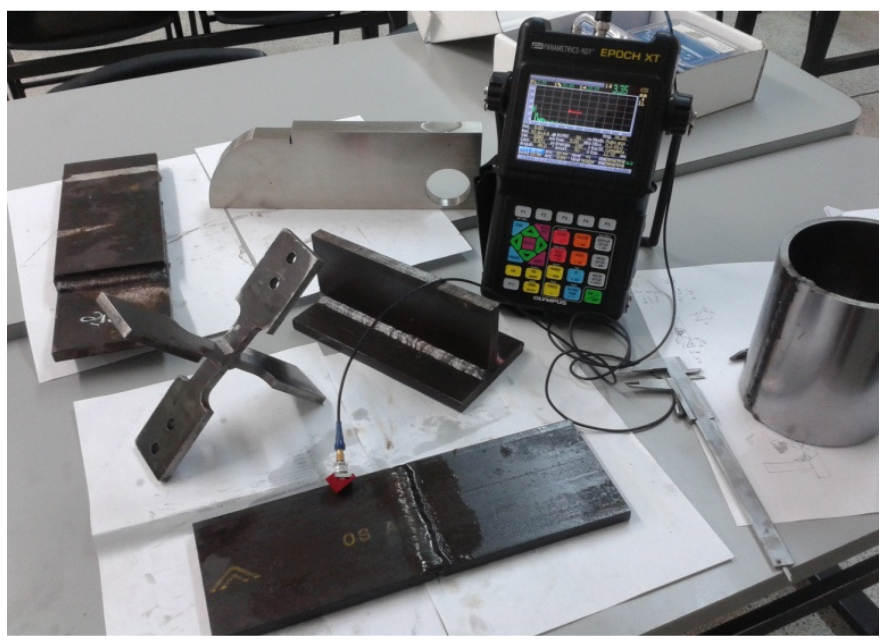

Figura 3. Equipamiento utilizado en la inspección ultrasónica.

\section{PROCEDIMIENTO EXPERIMENTAL}

Para realizar el procedimiento de caracterización de defectos en las uniones soldadas es necesario preparar el equipo, a continuación se describe el procedimiento.

- Preparación de superficies a inspeccionar: Las probetas deben ser limpiadas de tal forma que no queden residuos que puedan interferir en la medición o rayar los palpadores del equipo de ultrasonido.

- Procedi miento de calibración inicial de ultrasonido: Utilizando un bloque de calibración IIW Type I, se procede a realizar la calibración de la velocidad de propagación del sonido en el medio y se definen los ajustes del emisor y del filtro, esto con el fin de crear una imagen nítida del A-Scan, para el palpador angular de $45^{\circ}$ y $5 \mathrm{MHz}$.. En la Figura 4 se indica este procedimiento.

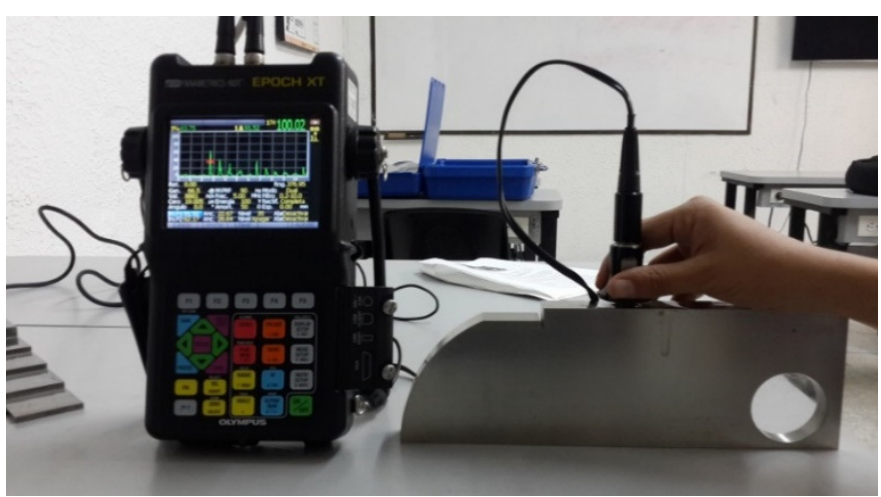

Figura 4. Procedimiento de calibración inicial para la inspección ultrasónica.

- Con el propósito de caracterizar las diversas tipologías de defectos en soldadura se realiza la inspección sobre los especímenes fabricados, en general los procedimientos se encuentran cobijados bajo las Normas de referencia ASTM SE-797 Standard Practice for Measurement By Manual contact Ultrasonic Method y ASTM A-435 Standard Specification for straight Beam Ultrasonic examination of steel Plates.

\section{RESULTADOS Y DISCUSIONES}

La naturaleza de una discontinuidad presente en una soldadura puede ser determinada al observar el comportamiento de las indicaciones sobre la pantalla del A Scan cuando el transductor es manipulado y es interceptado el haz ultrasónico por la discontinuidad.

A continuación en la Tabla 1 se muestran los resultados producto de la inspección por Rayos X correspondiente a diferentes probetas inspeccionadas. 


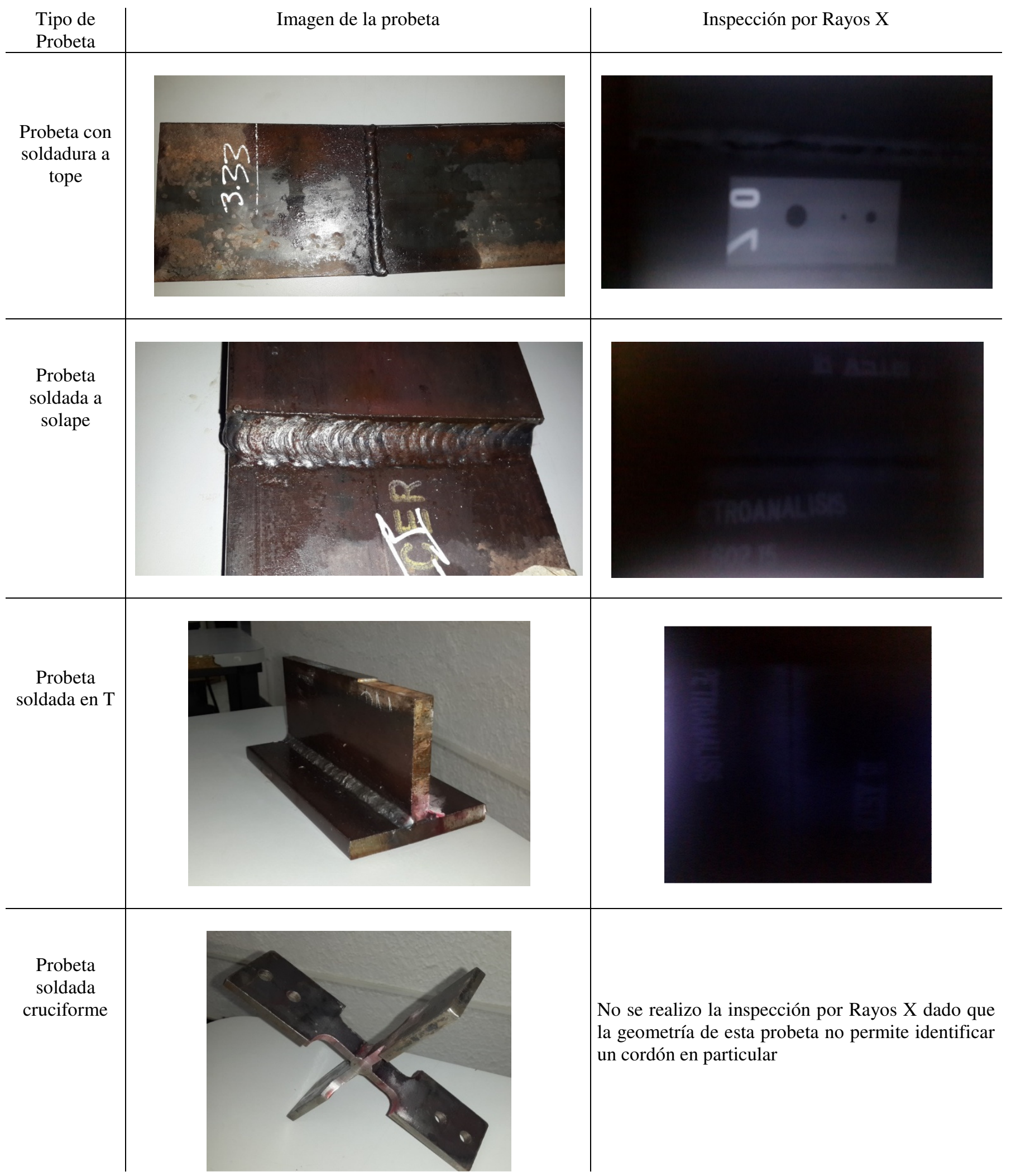

Tabla 1. Inspección por Rayos X. 


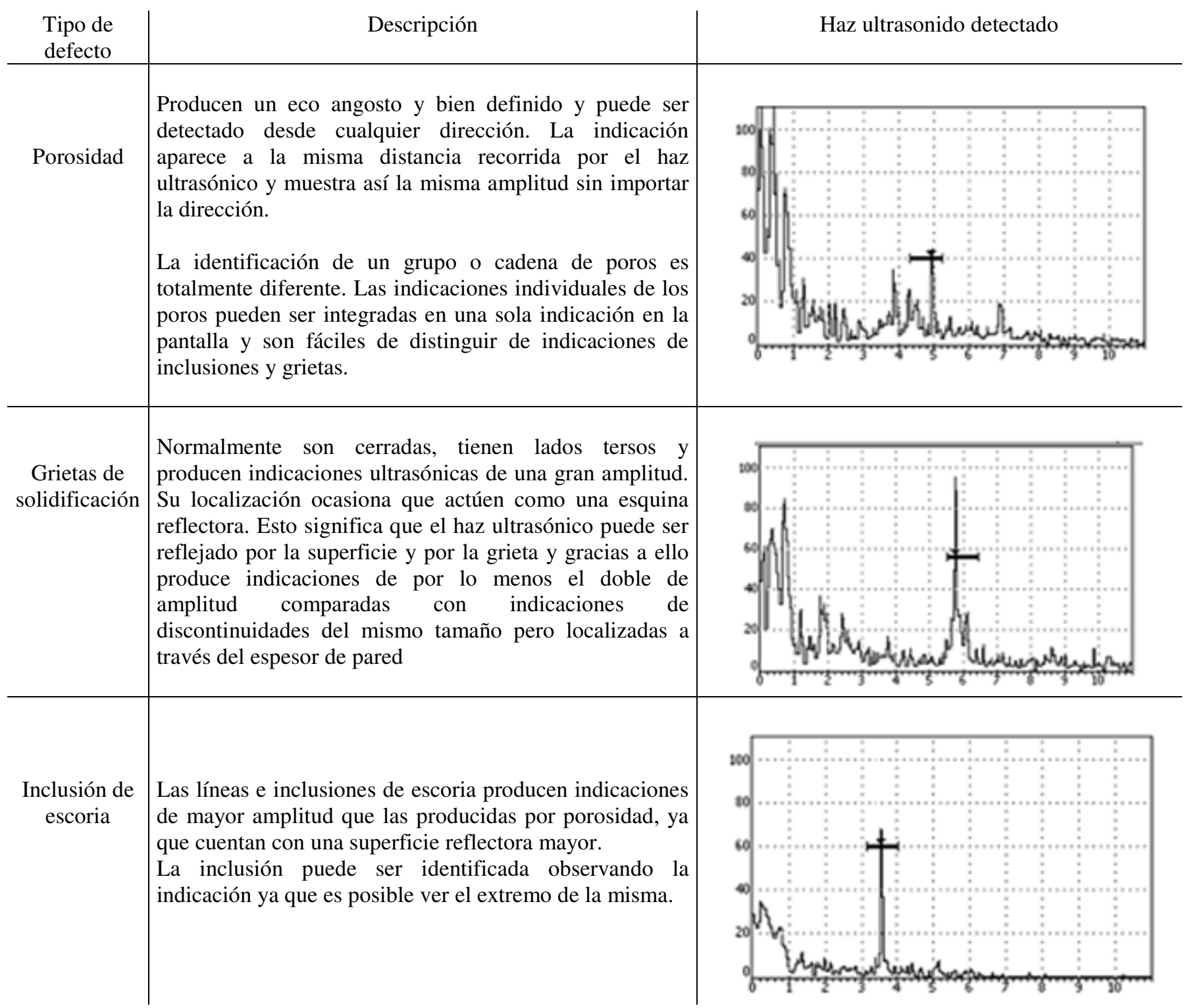

Tabla 2. Discontinuidades detectadas por ultrasonido. Adaptado de [9].

En la Tabla 2 se indican los resultados producto de la inspección por ultrasonido Scan A, correspondiente a diferentes probetas inspeccionadas.

Utilizando la técnica de Rayos $\mathrm{X}$ es posible corroborar la identificación de los defectos producto de la inspección ultrasónica, acá se distinguen principalmente tres tipos de discontinuidades, se establece que las porosidades son inherentes al proceso de soldadura y están presentes en mayor o menor intensidad en todas las probetas inspeccionadas, sin embargo y conforme a los códigos de soldadura, generalmente son aceptadas y no interfieren en gran medida con la integridad estructural de las uniones. Los defectos tipo grieta de solidificación y algunas inclusiones de escoria generalmente pueden llegar a comprometer la integridad de la unión estructural, los códigos de soldadura y ultrasonido definen en función de la sensibilidad al momento de la calibración del equipo de ultrasonido, la aceptación o no de estos en función del tamaño del haz ultrasonido.

Generalmente se logran distinguir las discontinuidades que mayor injerencia tienen en la integridad estructural de una unión soldada por el tamaño y forma del haz ultrasonido, para ello es necesario realizar con el palpador desplazamirntos hacia adelante y hacia atrás, realizando un movimiento en "zigzag" este movimiento permite detectar todos los defectos inclinados, en ocasiones es necesario realizar una verificación posterior desde lados opuestos al cordón inspeccionado o con 
palpadores angulares con ángulos diferentes por ejemplo $60^{\circ}$, $70^{\circ}$.

\section{CONCLUSIONES}

La inspección por ultrasonido requiere de un conocimiento previo en la técnica y de condiciones operacionales que pueden diferir en función del material o las condiciones ambientales en las cuales se localiza la pieza a evaluar.

Se identificó diferentes tipos de defecto, y se han comparado los haces ultrasónicos obtenidos por el equipo de ultrasonido con los encontrados en la literatura especializada para este tipo de ensayos.

El ensayo de Rayos X, permite comparar los resultados obtenidos con los del equipo de ultrasonido, esto permite afirmar que el ensayo de ultrasonido es una técnica confiable y aceptada para la detección de defectos internos en soldaduras.

\section{AGRADECIMIENTOS}

El autor expresa su agradezco a la Dirección de Investigaciones de la Universidad de Ibagué y al estudiante Hector Lizarralde del programa de Ingeniería Mecánica, por el apoyo suministrado para el desarrollo de esta investigación.

\section{REFERENCIAS}

[1]. G.F. Marlone, "Materiales metálicos", Tesis Universidad Tecnológica Nacional, Buenos aires, 2004.

[2]. J. Corre, "Apuntes Técnicos para todos". [Online]. Available:http://juliocorrea.files.wordpress.com/2007 /10/pruebas-no-destructivas.pdf (Octubre de 2007 ).

[3]. W. Olarte, M. Botero, "La detección de ultrasonido: una técnica empleada en el mantenimiento predictivo," Scientia et Technica, Año XVII, No 47, pp. 230-233. Abril de 2011.

[4]. ICONTEC Guía para la inspección de soldadura mediante ensayos no destructivos, NTC 2120, Julio, 1994.

[5]. E. Santos De La Cruz, N. Cancino Vera, J. Yenque Dedios, D. Ramírez Morales, M. Palomino Pérez, "El ultrasonido y su aplicación," Revista de la Facultad de Ingeniería Industrial. Vol. (8) 1, pp. 2528, 2005.

[6]. L. Barrera, M. Coronel, "Desarrollo y procedimientos para la utilización de tecnicas de ensayos no destructivos con ultrasonido para inspección de tuberías", pp. 1-7 Recuperado el 2014 2011
[7]. C. Rimoldi, L. Mundo, "Ensayos no destructivos", pp 54-78, 2012.

[8]. O. Araque, N. Arzola, "Estado del arte sobre la integridad estructural de uniones soldadas y modelos de propagación de grietas para la gestión de vida en estructuras," Ingeniare. Revista chilena de ingeniería, vol. $21 \mathrm{~N}^{\mathrm{o}}$ 2, pp. 279-292. 2013

[9]. I. Leon, C. Russo, "Curso Nivel I y II Ultrasonido", [Online]. Available: http://es.scribd.com/doc/105822227/Curso-deUltrasonido-Nivel-I-y-II\#scribd(2012, Sep.) 